\title{
D'abord, la mauvaise nouvelle ...
}

L

es médecins apprennent à annoncer une mauvaise nouvelle en fonction de leur interlocuteur : le deuil subit, le diagnostic terminal, le résultat d'examen de mauvais augure. L'impulsion paternaliste d'éviter aux patients les vérités horribles est chose du passé. On conseille aussi aux médecins d'admettre l'erreur qui peut se produire et d'expliquer rapidement et franchement ce qui tourne mal. Ces normes de divulgation reposent sur la prémisse selon laquelle les patients ont le droit d'être informés de leur propre situation.

Cette entité collective qu'est le public n'a-t-elle pas aussi le «droit de savoir»? En santé publique, il existe malheureusement une tradition de longue date consistant à dévoiler au compte-gouttes la vérité à un public auquel on attribue le peu d'intelligence et la panique facile d'un troupeau. La diffusion du risque est un art subtil, c'est certain : comment persuader le public d'adopter les changements de comportement qui s'imposent sans provoquer une anxiété indue ou des comportements compensatoires qui donnent naissance à de nouveaux risques? De l'encéphalopathie spongiforme bovine au virus du Nil occidental en passant par les BPC dans le lait maternel, la diffusion d'un risque doit faire l'objet de sa propre évaluation' Une stratégie que l'on songe depuis peu à envisager consiste à prévenir le public seulement des risques importants qu'il peut vraiment éviter en prenant les mesures voulues.

Certains voudraient nous faire croire que le public n'avait pas besoin d'être informé de l'éclosion de diarrhée associée à Clostridium difficile (DACD) dans des hôpitaux de Montréal et d'ailleurs. Lorsque notre journal a annoncé la nouvelle ${ }^{2}$, un administrateur d'hôpital et le ministre de la Santé du Québec ont déclaré à la presse que l'infection par $C$. difficile était une question hospitalière qui n'avait rien d'extraordinaire. Le ministre a déclaré que son ministère était au courant depuis quelque temps - et que tout ce qui avait changé, c'est que les reportages suscitaient l'inquiétude.

Cette nonchalance est ahurissante, mais il est vrai que le reportage sur le $C$. difficile comporte deux éléments. D'abord, il porte sur les données probantes indiquant qu'il se passe quelque chose de nouveau et d'inquiétant : il semble que les taux de DACD sont à la hausse et que la maladie devient plus virulente ${ }^{3}$. À cet égard, les enjeux et la façon de procéder sont clairs, sinon simples. Il faut améliorer la surveillance et la déclaration des maladies infectieuses nosocomiales, instaurer la collaboration entre les services de contrôle des infections dans les hôpitaux et le secteur de la santé publique, rationaliser l'utilisation des antibiotiques et tenir compte d'autres facteurs de risque possibles (comme les inhibiteurs de la pompe à protons) ${ }^{4}$. Il faut améliorer l'hygiène dans les hôpitaux et mettre l'infrastructure à niveau à cette fin ${ }^{3,5,6}$. Il faut effectuer davantage de recherches, y compris établir la cartographie génétique de l'organisme et la comparer avec des sous-types antérieurs.

Le deuxième volet de la question porte sur la divulgation au public. À cet égard, un changement d'attitude s'impose. Les hôpitaux sont financés par l'Etat et doivent servir le public. Il s'ensuit que le public a le droit de savoir ce qui se passe dans ces établissements, à la fois en général et parfois en particulier. Combien de Canadiens connaissent leur risque de contracter une infection à l'hôpital - risque que l'Institut canadien d'information sur la santé évalue à un sur neuf à la suite d'une enquête récente? ? Des États américains, à commencer par l'Illinois l'année dernière, imposent le suivi et la déclaration publique des taux d'infections nosocomiales afin d'améliorer les choix des consommateurs et de produire une incitation au changement.

Les données sur $C$. difficile dans les hôpitaux du Canada sont imparfaites - mais pour être efficaces, les interventions en santé publique ne peuvent attendre des données parfaites. On n'a pas le temps de laisser la nounoucratie s'imposer et aucune excuse pour le faire ${ }^{8}$. Les patients qui savent qu'ils devront faire face à une telle éclosion peuvent faire des choix plus éclairés au moment de décider où, si et quand subir une intervention chirurgicale élective. Les patients, le personnel et les visiteurs dans les hôpitaux pourraient faire davantage attention à la propreté des mains, à l'hygiène personnelle et au contact avec d'autres patients. Les patients qui quittent l'hôpital, et en particulier ceux qui sont à risque accru (c.-à-d. les personnes qui retournent chez elles en prenant des antibiotiques, les personnes âgées et les personnes immunodéprimées), pourraient être davantage à l'affût de signes avertisseurs de fièvre ou de diarrhée.

La situation comporte toutefois un troisième élément dont nous ne pouvons que présenter un aperçu. Il s'agit des médecins qui hésitent à se faire entendre lorsque des difficultés surgissent dans leurs hôpitaux. De ceux qui font des blagues de mauvais goût au sujet de la sécurité d'emploi lorsqu'ils pataugent publiquement dans la controverse scientifique. Des cliniciens et des stagiaires dans les services qui, perturbés par les dimensions éthiques de l'exposition de patients à des risques non divulgués, ont fait des efforts pour lancer une discussion franche sur C. difficile. Dans un article portant sur de mauvaises nouvelles, le muselage des inquiétudes exprimées aux premières lignes de la médecine est sans doute la pire nouvelle. - $7 A M C$

\section{Références}

1. Powell D, Leiss W. Mad cows and mother's milk: the perils of poor risk communication. Montréal et Kingston : Presses universitaires McGill-Queen's; 1997.

2. Eggertson L, Sibbald B. Hospitals battling outbreaks of C. difficile. CMAJ 2004;171(1):19-21.

3. Valiquette L, Low DE, Pépin J, McGeer A. Clostridium difficile infection in hospitals: a brewing storm. CMA7 2004;171(1):27-9.

4. Dial S, Alrasadi K, Manoukian C, Huang A, Menzies D. Risk of Clostridium difficile diarrhea among hospital inpatients prescribed proton pump inhibitors: cohort and case-control studies. CMA7 2004;171(1):33-8.

5. Louie TJ, Meddings J. Clostridium difficile infection in hospitals: risk factors and responses [éditorial]. CMAf 2004;171(1):45-6.

6. Loo VG, Libman MD, Miller MA, Bourgault AM, Frenette CH, Kelly M, et al; Clostridium difficile Associated Diarrhea Clinical Study Investigators Group. Clostridium difficile: a formidable foe [éditorial]. CMA7 2004;171(1):47-8.

7. Institut canadien d'information sur la santé. Les soins de santé au Canada, 2004 Ottawa: L'Institut; 2004.

8. De la nounoucratie à la divulgation : le rapport de l'enquête sur l'ESB [éditorial]. FAMC 2001;164(2):167. 